\title{
Seguimiento audiológico del recién nacido de muy bajo peso
}

\author{
Hearing assesment in extremely low birth weight infants
}

Mariela Torrente $\mathrm{A}^{1}$, Javiera Retamal M², Marcia Núñez T³.

\begin{abstract}
RESUMEN
Introducción: Los recién nacidos de muy bajo peso (RNMBP) pueden tener diversas deficiencias sensoriales como secuela de las patologías perinatales propias de esta población, entre ellas la hipoacusia sensorioneural (HSN).

Objetivos: El objetivo del presente trabajo es establecer la prevalencia de HSN en lactantes con el antecedente de ser RNMBP, es decir, con peso de nacimiento menor a 1500 gramos.

Material y método: Se incluyeron los pacientes con peso de nacimiento $<1500 \mathrm{gr}$, que nacieron en el Hospital San Juan de Dios (Santiago, Chile) entre el $1^{\circ}$ de enero de 1999 y el 31 de diciembre de 2004. A todos los pacientes se les realizó examen de potenciales auditivos evocados y evaluación por otorrinolaringólogo. Se definió como audición normal un umbral $\leq 40 \mathrm{dBHL}$.

Resultados: Ingresaron al seguimiento 147 pacientes, con una edad gestacional promedio de $30 \pm 2$ semanas y peso de nacimiento promedio de $1235 \pm 254 \mathrm{gr}$. De ellos 113 tuvieron umbral normal (76,9\%), con una latencia para la onda I de 1,78 $\pm 0,21 \mathrm{~ms}$, para la onda $V$ de 6,34 $\pm 0,29$ ms y para el intervalo l-V de 4,55 $\pm 0,26$ ms. En 16 pacientes se diagnosticó hipoacusia bilateral (11\%).En seis de ellos se confirmó el diagnóstico de HSN bilateral, lo que equivale al $4 \%$ de la población incluida en el estudio.

Conclusión: La prevalencia de un 4\% de HSN en el grupo estudiado coincide con la literatura internacional. Los potenciales auditivos evocados en la detección de HSN en RNMBP constituyen un examen confiable con una especificidad del $90 \%$ y una sensibilidad del $100 \%$.

Palabras clave: Hipoacusia, recién nacido bajo peso, pesquisa, prematuro, potenciales auditivos.
\end{abstract}

\section{ABSTRACT}

Introduction. Very low birth weight newborns (ELBW) may present with different sensory deficiencies as a result of perinatal pathologies characteristic to this group; among them, sensorineural hearing loss (SNHL).

Aims. The aim of the present study was to establish the prevalence of sensorineural hearing loss in ELBW (birth weight of less than 1500g) infants in a public hospital.

Materials and Methods. Premature infants born with a birth weight $<1500 \mathrm{~g}$, born at the Hospital San Juan de Dios (Santiago, Chile) between January 1 st 1999 and December 31st 2004 were included. All patients were evaluated by auditory brainstem response, recording the hearing threshold, wave I and V latencies, and I-V interval. A normal threshold was defined as less or equal to $40 \mathrm{~dB}$ hearing level.

\footnotetext{
Médico del Servicio de Otorrinolaringología, Hospital San Juan de Dios. Facultad de Medicina Universidad de Chile, Campus Occidente.

Alumna de la Escuela de Medicina Universidad de Chile

Fonoaudióloga del Servicio de Otorrinolaringología, Hospital San Juan de Dios
} 
Results. 147 patients were evaluated, with a gestational age of $30+-2$ weeks and a birth weight of $1235+-254 \mathrm{~g}$. Of these, 113 had a normal ABR threshold (76.9\%), with a wave I latency of $1.78+-0.21$, wave $V$ latency of $6.37+-0.29$, I-V interval $4.55+-0.26$. Sixteen patients had a bilateral threshold higher than $40 \mathrm{~dB}(11 \%)$. Of these patients, six had bilateral threshold elevation with an increase in I-V interval, suggesting central hearing impairment.

Conclusion. In the population of premature infants evaluated, the prevalence of bilateral SNHL was $4 \%$.

Key words. Hearing loss, low birth weight infant, premature, auditory screening.

\section{INTRODUCCIÓN}

La hipoacusia sensorioneural (HSN) bilateral es una patología presente en 2 a 5 de cada 1000 recién nacidos vivos ${ }^{1-3}$. Para el niño se traduce en una severa discapacidad y para la sociedad constituye la pérdida de un individuo económicamente productivo.

Su prevención es difícil ya que sólo $50 \%$ de los pacientes tiene factores de riesgo, tales como bajo peso de nacimiento $(<1.500$ g.), Apgar bajo, hiperbilirrubinemia, uso de ototóxicos, TORCH, malformaciones craneofaciales, antecedente de meningitis, antecedentes familiares de hipoacusia ${ }^{4}$. La edad promedio de diagnóstico de HSN se ha estimado en 30 meses, lo cual se traduce en un proceso de rehabilitación tardío. Yoshinaga-Itano y cols demuestran que si un niño sordo es diagnosticado e implementado con prótesis auditiva antes de los seis meses de vida, tendrá un desarrollo normal del lenguaje ${ }^{5}$. Así, nuestra meta debe ser la detección precoz de la hipoacusia, es decir, antes de los seis meses de vida.
La evaluación de audición en un lactante tradicionalmente se ha realizado mediante parámetros clínicos, tales como la respuesta al sonido (reflejo cócleo-palpebral), seguimiento de una fuente sonora. Sin embargo, estos métodos tienen una baja sensibilidad y especificidad. Lo óptimo es recurrir a exámenes objetivos de audición. Actualmente contamos con dos herramientas: Ios potenciales auditivos evocados de tronco cerebral (también conocidos por su sigla en inglés BERA) y las emisiones otoacústicas.

Los potenciales auditivos evocados de tronco cerebral (PAE) se realizan con el paciente en sueño fisiológico o sedado con hidrato de cloral. Se obtiene un registro de superficie donde se reconocen cinco ondas que se corresponden con determinadas estructuras anatómicas (Figura 1) ${ }^{6}$. La intensidad del estímulo se puede modificar generando un umbral de audición, lo que permite diagnosticar una hipoacusia cuando el umbral es mayor a $40 \mathrm{dBHL}$.

El registro también permite diferenciar el tipo de hipoacusia: de conducción o sensorioneural

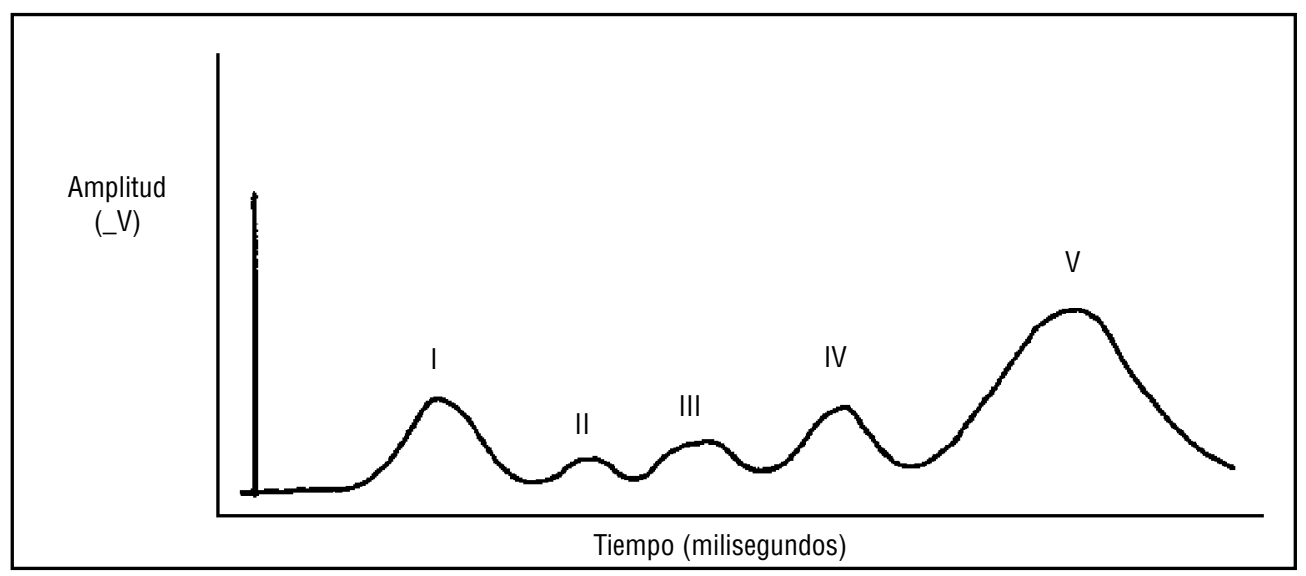

Figura 1. Curva de potenciales auditivos evocados de tronco cerebral y su relación anatómica. 


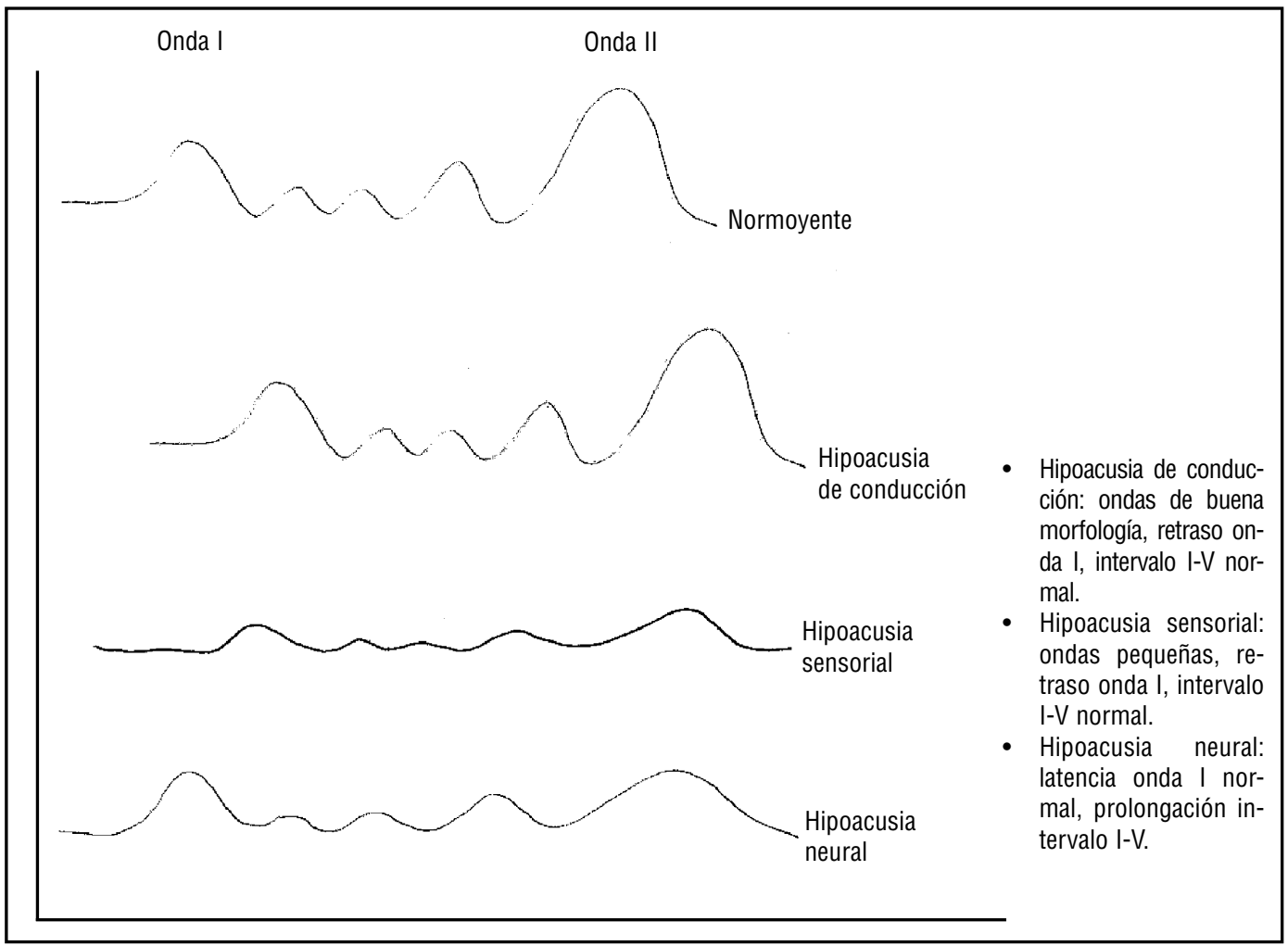

Figura 2. Diagnóstico diferencial de hipoacusia en examen de potenciales auditivos evocados.

(Figura 2). Para esta diferenciación se recurre a las latencias de las ondas que se obtienen en el trazado, particularmente las ondas I y $\mathrm{V}, \mathrm{y}$ el intervalo entre ambas. Sin embargo, el valor absoluto de estas latencias varía según la edad del paciente en estudio. Con respecto a la hipoacusia sensorial, el diagnóstico se puede confundir con hipoacusia de conducción ya que en ambas se prolonga la latencia de la onda I. Sin embargo, las ondas de un paciente con hipoacusia sensorial son de pobre morfología, y la impedanciometría surge como examen auxiliar.

EI PAE tiene una excelente correlación con los umbrales definitivos estimados por la audiometría ${ }^{7}$ y constituye el examen de elección en la evaluación de un lactante con sordera. Protocolos que utilizan potenciales secuenciados (se repite en los pacientes que fallan) tienen $3 \%$ de falsos positivos ${ }^{2}$.

En Chile, previo a la introducción del protocolo de Garantías Específicas en Salud (GES) en julio de 2005, existía un Programa Nacional de Seguimien- to del Recién Nacido Prematuro Extremo, desde el año 1999, que incluía la evaluación audiológica en los primeros seis meses de vida mediante potenciales auditivos evocados.

El objetivo del presente trabajo es evaluar los resultados del seguimiento prospectivo de la cohorte. Los objetivos secundarios son establecer la prevalencia de hipoacusia sensorioneural bilateral en la población en estudio, obtener valores de referencia para la latencias de ondas en el PAE y describir las características del PAE como examen de pesquisa y diagnóstico en nuestra población.

\section{MATERIAL Y MÉTODO}

El presente trabajo tiene un diseño prospectivo, de seguimiento de casos, con análisis descriptivo. Todos los RNMBP dados de alta de la Unidad de Neonatología del Hospital San Juan de Dios fueron citados a seguimiento al Policlínico de Alto Riesgo, 
dependiente de dicha unidad. El pediatra solicitó el examen PAE que se realizó en el Instituto de Rehabilitación Infantil TELETON. Con el resultado de dicho examen fueron ingresados en policlínico de seguimiento del Servicio de Otorrinolaringología del Hospital San Juan de Dios. Todos los niños fueron evaluados por otorrinolaringólogo, se solicitó impedanciometría cuando se estimó necesario (sospecha de patología de oído medio). Si el examen clínico y el PAE son normales, los niños se citaron cada seis meses hasta los 4 años. Los pacientes fueron dados de alta una vez realizada una audiometría, cuyo informe fuese normal. La audiometría se realizó en cabina insonorizada, con un equipo marca Madsen modelo Orbiter, con fonos. Para este examen se consideró normal un umbral menor a $20 \mathrm{~dB}$.

Los potenciales auditivos fueron realizados con el paciente bajo sedación con hidrato de cloral (30$50 \mathrm{mg} / \mathrm{Kg}$ ), con un equipo marca Medelec modelo V. Se aplicaron clicks de rarefacción en forma separada en ambos oídos con una intensidad de 80 $\mathrm{dBHL}$, con enmascaramiento contralateral de 30 dB. Se promedió la respuesta a 1024 clicks obteniendo una curva. La intensidad del estímulo se disminuyó hasta que desapareció la onda V. Se consideró audición normal con umbral $\leq 40 \mathrm{dBHL}$.

Se incluyeron en la presente evaluación los pacientes con fecha de nacimiento entre el $1^{\circ}$ de enero de 1999 y el 31 de diciembre de 2004 que ingresaron a seguimiento en el policlínico de Otorrinolaringología.

\section{RESULTADOS}

Durante el período en estudio nacieron en la maternidad del Hospital San Juan de Dios 24.764 recién nacidos, de los cuales 392 fueron RNMBP, lo que equivale al $1,6 \%$ de los partos. De estos neonatos sobrevivieron 274 (70\%). El peso promedio fue de $1228 \pm 254,8 \mathrm{~g}$, y la edad gestacional promedio fue de $30,2 \pm 2,5$ semanas.

Ingresaron a seguimiento en policlínico de otorrinolaringología 147 pacientes lo que equivale al $53,6 \%$ del universo potencial. La edad gestacional promedio fue de $30 \pm 2$ semanas y el peso de nacimiento promedio fue de $1235 \pm 254 \mathrm{~g}$.

Del período neonatal se registró Apgar al primer minuto (mediana de 7, rango 1-9), Apgar a los
5 minutos (mediana 9 , rango 1-10), bilirrubinemia (mediana $10 \mathrm{mg} / \mathrm{dl}$, rango 7-18,8) y ventilación mecánica (mediana 0 días, rango 0-70). En 111 pacientes se usaron ototóxicos, principalmente aminoglicósidos y diuréticos de asa. La evaluación audiológica se realizó en promedio a los ocho meses de edad corregida.

A toda la muestra se le realizó PAE. Ciento trece pacientes tuvieron un examen normal, es decir, con umbral $\leq 40 \mathrm{dBHL}(76,9 \%)$ y 34 tuvieron un umbral auditivo alterado $(23,1 \%)$ en uno 0 ambos oídos. Dieciocho pacientes presentaron elevación del umbral auditivo en forma unilateral, de los cuales siete tienen actualmente audiometría normal. El resto continúa en seguimiento monitorizando el desarrollo del lenguaje.

Dieciséis pacientes presentaron elevación del umbral auditivo en forma bilateral, lo que correspondió al $11 \%$ de la muestra. La evolución de estos pacientes mostró:

- Audiometría normal en 9 casos.

- Un paciente se le diagnosticó patología de oído medio (OME) que explicaba su hipoacusia.

- Hipoacusia Sensorioneural:

- Prolongación de la latencia de la onda I con conservación del intervalo I-V en tres casos, dos de ellos son usuarios de audífonos actualmente.

- Un paciente tenía trazado sin ondas, actualmente es usuaria de audífonos

- Un paciente tenía latencias dentro de límites normales, con umbral de 45 y $55 \mathrm{dBHL}$.

- Un paciente presentó prolongación de la latencia de la onda I y del intervalo I-V, sin embargo, se perdió de seguimiento.

Por lo tanto, del total de la muestra, en seis pacientes se confirmó el diagnóstico de hipoacusia sensorioneural bilateral, lo que correspondió al 4\% de la muestra incluida (Tabla 1).

Los pacientes portadores de HSN bilateral presentaron diversos factores de riesgo. Sus características epidemiológicas pueden verse en la Tabla 2.

Para el cálculo de las latencias se utilizaron los trazados con umbral auditivo normal. Es así como se obtuvieron valores promedio de latencia para la onda I de 1,78 $\pm 0,21 \mathrm{~ms}$, para la onda $V$ de 6,37 $\pm 0,29 \mathrm{~ms}$, y para el intervalo I-V de 4,55 $\pm 0,26 \mathrm{~ms}$ (Tabla 3). 


\section{DISCUSIÓN}

Este estudio tiene la riqueza de aportar información sobre un grupo beneficiario del sistema público de salud de la Región Metropolitana. A la inversa, tiene la limitación de no haber conseguido una cobertura total de los niños allí atendidos. Lograr esto es prácticamente imposible, por la movilidad de la población y las dificultades para acceder a la atención en el caso de los niños de procedencia rural. Por lo demás, la prevalencia encontrada (4\%) concuerda con los datos de la literatura

Tabla 1. Resultado de examen de potenciales auditivos evocados de tronco cerebral en recién nacidos de muy bajo peso ( $n: 147$ )

\begin{tabular}{|lc|}
\hline & Frecuencia \\
\hline PAE normal & 113 \\
PAE alterado & 34 \\
- Unilateral & 18 \\
- Audiometría normal & 7 \\
- OME & 1 \\
- HSN & $10(6,8 \%)$ \\
- Bilateral & 16 \\
- Audiometría normal & 9 \\
- OME & 1 \\
- HSN & $6(4 \%)$ \\
\hline
\end{tabular}

extranjera ${ }^{8}$, de modo que es posible suponer que el error de ésta no debe alcanzar magnitudes importantes.

Se ha descrito en la literatura hipoacusia de instalación tardía, entre los 12 y 24 meses de vida, en menores con factores de riesg $0^{9}$. Por esta razón en nuestra institución el seguimiento se prolonga hasta los 4 años de edad, independiente del resultado del PAE. En estos cinco años de funcionamiento no hemos tenido casos de hipoacusia de instalación tardía.

Entre las posibles etiologías de la HSN en estos pacientes podemos mencionar los períodos de hipoxia, hiperbilirrubinemia, uso de medicamentos ototóxicos (fundamentalmente amikacina y furosemida), TORCH, meningitis ${ }^{10}$. Lo más frecuente de observar es una asociación de ellos.

El examen de potenciales auditivos evocados efectuado en el Instituto de Rehabilitación Infantil TELETON es confiable $(100 \%$ de correlación con audiometría para el diagnóstico de hipoacusia) ${ }^{13}$. De los resultados obtenidos en el presente trabajo, al utilizar potenciales auditivos evocados en la detección de HSN en RNMBP se obtiene una especificidad de $90 \%$ y sensibilidad de $100 \%$, no hubo falsos negativos y los falsos positivos fueron 10\% (Tabla 4). Al evaluar un examen de pesquisa es importante el valor predictivo de la prueba positiva (VPPP), que en este caso corresponde a 0,44 . Este resultado tan pobre del VPPP se explica

Tabla 2. Antecedentes perinatales de pacientes RNMBP portadores de hipoacusia sensorioneural bilateral (Paciente número 3 no se disponen de más datos, paciente número 6 se perdió de seguimiento)

\begin{tabular}{|c|c|c|c|c|c|c|c|c|}
\hline & $\begin{array}{c}\text { EG } \\
\text { (semanas) }\end{array}$ & $\begin{array}{l}\text { PN } \\
(g)\end{array}$ & $\begin{array}{l}\text { Apgar } \\
1 \mathrm{MIN} \text {. }\end{array}$ & $\begin{array}{l}\text { Apgar } \\
5 \text { MIN. }\end{array}$ & $\begin{array}{l}\text { Vent. } \\
\text { Mec. } \\
\text { (días) }\end{array}$ & $\begin{array}{l}\text { Bilirrubinemia } \\
\text { Máxima } \\
(\mathrm{mg} / \mathrm{dl})\end{array}$ & Ototóxicos & $\begin{array}{r}\text { Umbral } \\
\text { auditivo } \\
\text { (dBHL) }\end{array}$ \\
\hline Paciente1 & 29 & 820 & 6 & 8 & 0 & 15,4 & Sí & 80 bilateral \\
\hline Paciente2 & 30 & 1460 & 8 & 8 & 0 & 6,0 & Sí & $\begin{array}{l}70 \text { OD } \\
6001\end{array}$ \\
\hline Paciente 3 & 33 & 720 & & & & & & $\begin{array}{l}80 \text { OD } \\
7001\end{array}$ \\
\hline Paciente 4 & 26 & 760 & 5 & 6 & 48 & 11,7 & Sí & $\begin{array}{l}80 \text { OD } \\
7001\end{array}$ \\
\hline Paciente 5 & 32 & 1660 & 8 & 9 & 0 & 17,6 & Sí & Registro plano \\
\hline
\end{tabular}

EG: edad gestacional. PN: peso de nacimiento. 
Tabla 3. Promedio de latencias de onda en examen de potenciales auditivos evocados, en lactantes con antecedente de RNMBP con audición normal (umbral $<40 \mathrm{dBHL}$ )

\begin{tabular}{|cccc|}
\hline $\begin{array}{c}\text { Onda I } \\
(\mathrm{ms}) \\
- \pm \mathrm{ds}\end{array}$ & $\begin{array}{c}\text { Onda V } \\
(\mathrm{ms}) \\
- \pm \mathrm{ds}\end{array}$ & $\begin{array}{c}\text { Intervalo I-V } \\
(\mathrm{ms}) \\
- \pm \mathrm{ds}\end{array}$ \\
\hline Latencia & $1,81 \pm 0,22$ & $6,37 \pm 0,36$ & $4,56 \pm 0,34$ \\
\hline
\end{tabular}

por la baja prevalencia de la enfermedad, y porque el examen PAE también detecta hipoacusia de conducción, patología mucho más frecuente que la hipoacusia sensorioneural. Sin embargo, los métodos disponibles para evaluar audición en lactantes no permiten diferenciar estas patologías con un examen aislado; por ello es necesario realizar exámenes complementarios. El valor predictivo negativo de la prueba (VPPN) es de 1.0 ya que no hubo falsos negativos.

Los valores de latencias obtenidos para las ondas de los PAE constituye la primera comunicación a nivel nacional. Puede utilizarse como referencia en recién nacidos MBP a los 6 meses de edad corregida. Las cifras obtenidas son similares a las reportadas para lactantes sin antecedentes mórbidos, de entre seis y nueve meses de edad ${ }^{14,15}$.

La vía auditiva ya se encuentra anatómicamente viable a las 26 semanas de edad gestacional, su desarrollo posterior se relaciona con su funcionalidad. Por ello en un recién nacido de 30 semanas se puede obtener un registro en
PAE pero con una latencia mayor de las ondas que lo que se obtiene en un recién nacido de término ${ }^{16}$. Este fenómeno se informa como falta de maduración de la vía auditiva. Cabe destacar que cuando se evalúa un recién nacido buscando hipoacusia, Io que más interesa es la presencia de ondas y cuál es el umbral de audición, fenómeno que no tiene relación con la maduración de la vía. Por lo tanto, en el informe de un examen de potenciales auditivos se debe prestar atención al umbral.

Una vez hecho el diagnóstico de hipoacusia, son importantes las latencias de las ondas. Y es en esta etapa de la evaluación del examen donde debe haber valores normados para las distintas poblaciones a evaluar: pretérminos, de término, lactantes, adultos.

En el seguimiento se logró incorporar a sólo $53,6 \%$ de los pacientes dados de alta de la Unidad de Neonatología. Este valor es muy pobre para un programa de pesquisa, que debiese incluir más del $95 \%$ de la población objetiva. La baja adherencia a los controles se explica por diversos factores: ruralidad, mortalidad posterior al alta, salida del país, colocación en casas de adopción o centros de pacientes crónicos.

\section{Agradecimientos}

Dra. María Teresa Henríquez, médico de la Unidad de Neonatología del Hospital San Juan de Dios, por su colaboración en la recolección de datos; y al Dr. Nelson Vargas, Jefe de Servicio Pediatría Hospital San Juan de Dios, por su revisión crítica del manuscrito.

Tabla 4. Tabla $2 \times 2$ para potenciales auditivos evocados (PAE) como prueba diagnóstica. Se consideró valor por oído estudiado

\begin{tabular}{|lccc|}
\hline & HSN confirmada & HSN descartada & Total \\
\hline PAE alterado & 22 & 28 & 50 \\
PAE normal & 0 & 244 & 244 \\
Total & 22 & 272 & 294 \\
\hline
\end{tabular}

Sensibilidad del examen para detectar hipoacusia: 100\%. Especificidad del examen para hipoacusia: $90 \%$ VPPP: 1. VPPN: 0,44 


\section{BIBLIOGRAFÍA}

1. Finitzo T, Albright K, o'Neal J. The newborn with hearing loss: Detection in the nursery. Pediatrics 1998; 102 (6): 1453-9.

2. Mason JA, Herrmann KR. Universal infant hearing screening by automated auditory brainstem response measurment. Pediatrics 1998; 101 (2): 221-8.

3. Vohr BR, Carty LM, Moore Pe, Letourneau K. The Rhode Island hearing assessment program. Experience with statewide hearing screening (1993-1996). J Pediatr 1998; 133 : 353-7.

4. Joint Committee on Infant Hearing 1994 Position Statement. Pediatrics 1995, 95: 152-6.

5. Yoshinaga-Itano C, Sedey Al, Coulter DK, Mehl AL. Language of early and later-identified children with hearing loss. Pediatrics 1998; 10 (5): 1161-71.

6. Biacabe B, Chevallier JM, Avan P, Bonfils P. Functional anatomy of auditory brainstem nuclei: application to the anatomical basis of brainstem auditory evoked potentials. Auris Nasus Larynx 2001; 28: 85-94.

7. Kileny PR, Magathan MG. Predictive value of $A B R$ in infants and children with moderate to profound hearing impairment. Ear Hear 1987; 8: 217-21.

8. Wood nS, Marlow N, Costeloe K, Gibson at, Wilkinson A. Neurologic and developmental disability after extremely preterm birth. $N$ Engl J Med 2000; 343: 378-84.

9. Robertson CM, Tyebkhan JM, Hagler ME, Cheung PY, Pelowski A, Etches PC. Late-onset, progressive sensorineural hearing loss after severe neonatal respiratory failure. Otol Neurotol 2002 May; 23(3): 353-6.

10. Marlow ES, Hunt LP, Marlow N. Sensorineural hearing loss and prematurity. Arch Dis Child Fetal Neonatal Ed 2000, 82 (2): F141-144

11. Amatuzzi MG, Northrop C, Liberman C, Thornton A, Halpin C, Herrmann B et als. Selective inner hair cell loss in premature infants and cochlea pathological patterns from neonatal intensive care unit autopsies. Arch Otolaryngol Head Neck Surg 2001; 127: 629-36.

12. Valkama AM, Laitakari KT, Tolonen EU, Väyrynen MRH, VAINIONPÄ̈̈ LK, KoIvisto ME. Prediction of permanent hearing loss in high-risk preterm infants at term age. Eur $J$ Pediatr 2000, 159:459-64.

13. Torrente M, Arancibia M, Poblete $C$, Leyton J, Núñez M, Corssen C et als. Evaluación del seguimiento auditivo del recién nacido prematuro extremo en el Hospital San Juan de Dios. Rev otorrinolaringol cir cab-cuello 2001; 61: 31-6.

14. Gorga MP, Kaminski JR, Beauchaine KL, Jesteadt W, NeELY ST. Auditory brainstem responses from children three months to three years of age: Normal patterns of response II. J Speech Hear Res 1989 Jun; 32(2): 281-8.

15. Katz J. Auditory brainstem response: Threshold estimation and auditory screening. En: Handbook of Clinical Audiology. Williams and Wilkins, cuarta edición.

16. Quiñónez RE, Crawford MR. Electrophysiologic changes in preterm neonates: Auditory brain stem response and distortion product otoacoustic emissions. Ann Otol Rhinol Laryngol 1997; 106: 721-8. 